\title{
Composite detection rate as an upper gastrointestinal endoscopy quality measure correlating with detection of neoplasia
}

\author{
Marcin Romańczyk ${ }^{1,2}$ (D) Bartosz Ostrowski ${ }^{1} \cdot$ Tomasz Marek $^{1} \cdot$ Tomasz Romańczyk $^{2}$ • \\ Małgorzata Błaszczyńska ${ }^{3}$ Krzysztof Budzyń ${ }^{2,1} \cdot$ Maciej Bugajski ${ }^{1}$. \\ Mateusz Koziej ${ }^{4}$ - Maciej Kajor ${ }^{5}$ - Krzysztof Januszewski ${ }^{6}$ - Wojciech Zajęcki ${ }^{7}$. \\ Marek Waluga $^{1} \cdot$ Marek Hartleb $^{1}$
}

Received: 17 December 2020/Accepted: 15 April 2021/Published online: 2 May 2021

(C) The Author(s) 2021

\begin{abstract}
Background Esophagogastroduodenoscopy (EGD) is commonly used diagnostic method with no widely accepted quality measure. We assessed quality indicator-composite detection rate (CDR) - consisting of detection of at least one of the following: cervical inlet patch, gastric polyp and postulcer duodenal bulb deformation. The aim of the study was to validate CDR according to detection rate of upper gastrointestinal neoplasms (UGN).

Methods It was a multicenter, prospective, observational study conducted from January 2019 to October 2019. The endoscopic reports from 2896 symptomatic patients who underwent diagnostic EGD were analyzed. The EGDs were performed in three endoscopy units located in tertiary
\end{abstract}

Marcin Romańczyk

mromanczyk@sum.edu.pl

1 Chair and department of Gastroenterology and Hepatology, School of Medicine in Katowice, Medical University of Silesia, Medyków 14 Street, 40-752 Katowice, Poland

2 Endoterapia, H-T. Centrum Medyczne, Tychy, Poland

3 Endoscopy Unit, District Hospitals of Chorzów Trust, Chorzów, Poland

4 Department of Anatomy, Jagiellonian University Medical College, Cracow, Poland

5 Department of Pathomophology and Molecular Diagnostic, School of Medicine in Katowice, Medical University of Silesia, Katowice, Poland

6 Department of Pathomophology, Zakład Diagnostyki Mikroskopowej, Dr Krzysztof Januszewski, Ruda Śląska, Poland

7 Department of Pathomophology, District Hospitals of Chorzów Trust, Chorzów, Poland university hospital, private outpatient clinic and local hospital.

Results 64 UGNs were detected. The mean CDR was 21.9\%. The CDR correlated with UGN detection rate $(R=0.49, p=0.045)$. Based on CDR quartiles, operators were divided into group 1 with $\mathrm{CDR}<10 \%$, group 2 with CDR $10-17 \%$, group 3 with CDR $17.1-26 \%$, and group 4 with CDR $>26 \%$. Detection rate of UGN was significantly higher in the group 4 in comparison to group 1 (OR 4.4; $95 \%$ CI $2.2-9.0$ ). In the multivariate regression model, patient age, male gender and operator's CDR $>26 \%$ were independent risk factors of UGN detection (OR 1.03; 95\% CI $1.01-1.05$, OR 2; $95 \%$ CI $1.2-3.5$, and OR $5.795 \%$ CI $1.5-22.3$, respectively).

Conclusions The CDR is associated with the detection of upper gastrointestinal neoplasms. This parameter may be a useful quality measure of EGD to be applied in general setting.

Keywords Esophagogastroduodenoscopy ·

Gastrointestinal neoplasm · Quality indicator · Upper gastrointestinal tract

\section{Introduction}

Endoscopy is widely available diagnostic examination of great importance in detection of malignant and non-malignant gastrointestinal lesions. The number of annually performed esophagogastroduodenoscopies (EGDs) only in the U.S. is over 6 million [1]. Esophageal and gastric cancers are still significant health problems, as both diseases are within the top ten most common cancers, which led in 2018 to 1.3 million deaths worldwide [2]. Precise inspection of the upper gastrointestinal tract allows 
detecting premalignant conditions and early cancers that is connected with better survival [3].

Importance of EGD quality has been raised in several endoscopy guidelines [4-6], as $6.4 \%$ of esophageal cancers and $9.4 \%$ of gastric cancers may be missed in routine EGD $[7,8]$. In the above-mentioned guidelines, the quality indicators relate to pre-, intra- and post-procedural activities. Apart from minimum time of the entire examination and inspection time dedicated to Barrett's esophagus, no definite quality measure, such as adenoma detection rate or cecal intubation rate for colonoscopy, has been established for EGD [9]. At the same time, detection of the neoplasia in the upper gastrointestinal tract could not be treated as quality indicator because of its low prevalence. Therefore, the surrogate parameters should be used to test the endoscopists inspection quality. Recently, endoscopist biopsy rate (EBR) has been proposed as applicable quality measure [10]. This parameter has been validated for gastric cancer and in expert hands, was related to lower risk of missed lesions.

The aim of our study was to validate a novel quality metrics based on operator's perception, named "composite detection rate" (CDR). CDR is based on the detection at least one of three mostly benign lesions: esophageal inlet patch, gastric polyp and post-ulcer duodenal bulb deformation. These findings were chosen as localized in all three investigated segments during EGD, fairly stable and no too low prevalence, not influenced by definition and requiring careful examination and/or special maneuvers. We assumed that search for those lesions pushes investigator to maximize focus on complete and exact inspection of three segments of upper digestive tract. We also hypothesized that thorough examination resulting in higher CDR is related to the detection of neoplastic lesions of the upper digestive tract.

\section{Materials and methods}

\section{Study design}

It was a prospective, multicenter, observational study conducted from January 2019 to October 2019. The study was performed in three centers: endoscopy unit of tertiary university hospital (center A), private outpatient endoscopy clinic (center B) and endoscopy unit of district hospital (center C). The study was exempt from institutional board review (reviewed by Ethic Committee of Medical University of Silesia decision no KNW/0022/KB/235/18). The study was conducted in accordance with the Declaration of Helsinki.

One thousand subsequent adults form each site, who were found eligible for elective diagnostic EGD, were enrolled to the study. The EGDs were performed by gastroenterologists, internal medicine or general surgery specialist after completed endoscopic training. Operators were aware of being observed of their quality of performance but were not informed what findings were assessed. Urgent and therapeutic procedures were excluded. Patient enrollment flowchart is summarized in Fig. 1.The recorded parameters were:

- Patient data: sex, age, indication for endoscopy.

- Procedure information: in/outpatient procedure, sedation, type of endoscope, name of the operator, biopsies taken.

- Endoscopy report findings: esophageal inlet patch, reflux esophagitis, Barrett's esophagus, esophageal polyp, esophageal tumor, gastritis, gastric polyp, gastric peptic ulcer, gastric tumor, duodenal bulb post-ulcer deformation, duodenal ulcer, duodenal polyp, duodenal tumor.

- Pathology report: Barrett's esophagus, Barrett's esophagus related dysplasia and adenocarcinoma, squamous intraepithelial dysplasia and carcinoma, esophageal stromal and neuroendocrine tumor, gastric dysplastic lesions, gastric cancer, gastric lymphoma, gastric neuroendocrine tumor, gastric stromal tumor, duodenal adenoma, duodenal cancer, duodenal lymphoma, duodenal neuroendocrine tumor, and duodenal stromal tumor.

We excluded from assessment patients with incomplete EGD (e.g., upper gastrointestinal obstruction, examination intolerance, inadequate preparation) or history of surgery (esophageal, gastric or duodenal resections). Operators who performed less than 50 procedures were excluded from operator's assessment. Helicobacter pylori infection was diagnosed in case of positive rapid urease test or in histopathological examination.

CDR was calculated as the proportion of patients in whom at least one of the following lesions were detected: esophageal inlet patches, gastric polyp and post-ulcer duodenal bulb deformation. EBR was calculated as the proportion of examinations with at least one biopsy taken.

Main endpoint of this study was upper gastrointestinal neoplasm (UGN) detection rate calculated as proportion of patients whom at least of the following was detectedcancer, non-epithelial tumor and precancerous condition (adenoma, gastric dysplastic lesion, squamous intraepithelial neoplasm, dysplasia in the Barrett's esophagus).

\section{Statistical analysis}

The quantitative data were reported as mean \pm standard deviation (SD) or median/interquartile range, according to distribution status fit. For the qualitative data, frequencies 


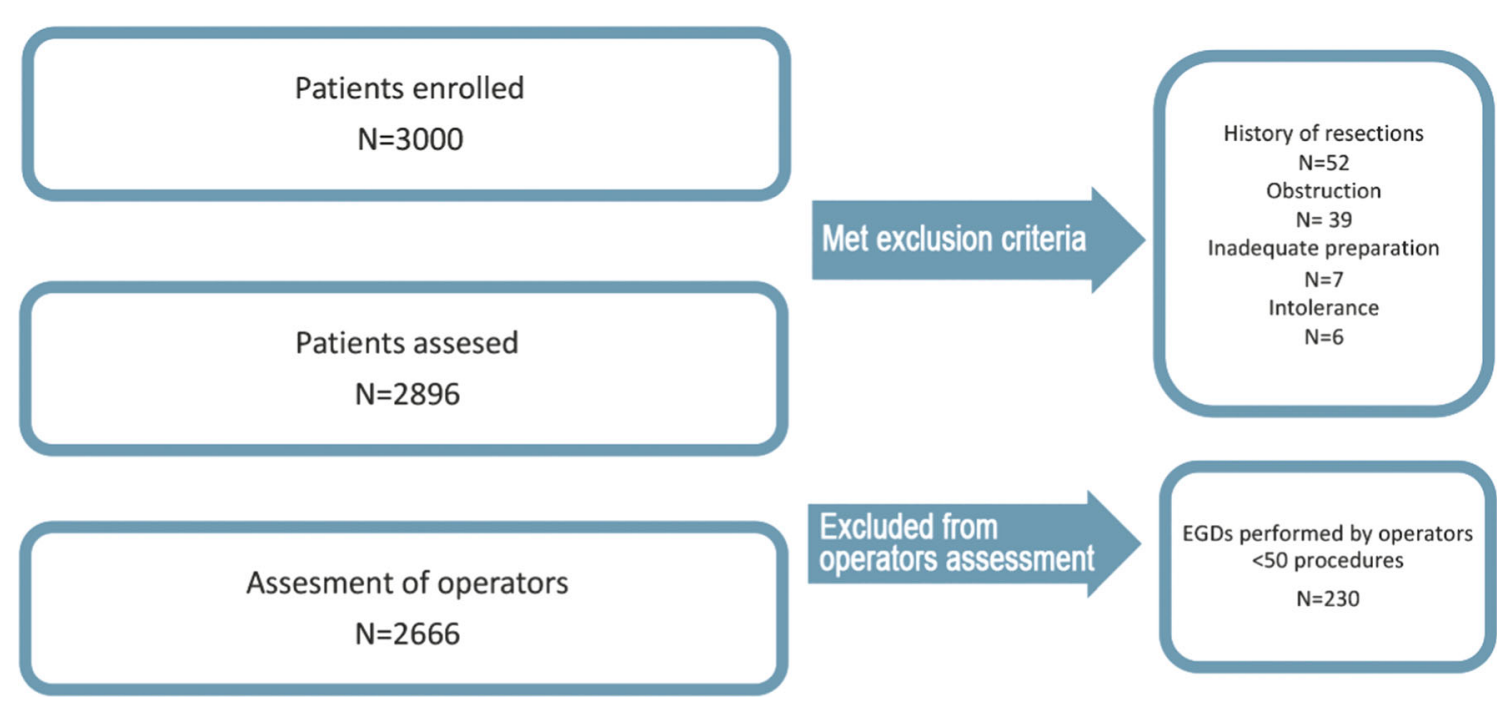

Fig. 1 Patient enrollment

and percentages were calculated. Odds ratios (ORs) and 95\% confidence intervals $(95 \% \mathrm{CI})$ for detection of UGN were calculated in relation to the baseline (group 1) for each of the groups $(2,3,4)$ based on CDR. Uni- and multivariate logistic regression analysis were performed to assess the risk factors of detecting UGN (dependent value). Also, Spearman correlation between CDR and EBR of each operator and UGN was performed. Comparisons were performed using $t$ test or Mann-Whitney test for two groups depending on normality. The qualitative variables were compared using the $\chi^{2}$ test of proportions for categorical variables with Bonferroni's correction if needed. The data were analyzed using StatSoft Statistica 13.0 PL for Microsoft Windows 10. The results with $p$ value $<0.05$ were considered as statistically significant. The sample size was estimated based on UGN prevalence that was known to be approximately $2.6 \%$ in the tertiary unit (based on the cohort of 1000 retrospectively assessed EGDs in the center A). The power analysis indicated that to detect correlation of $r=0.35$ utilizing a 2-sided test, $5 \%$ significance level test $(\alpha=0.05)$ with $80 \%$ power $(\beta=0.2)$, the required minimum sample size was 61 cases. Therefore, one thousand patients from each center was considered a representative sample.

\section{Results}

\section{General data}

Of 3000 eligible patients, 2896 were analyzed, (104 patients were excluded) - see Fig. 1. Demographic data and endoscopic findings are summarized in Table 1. Mean age of participants was 56.9 years. $66 \%$ were outpatients and $34 \%$ inpatients, $57.3 \%$ were female. Three most common indications for EGD were: gastroesophageal reflux disease symptoms (13.2\%), dyspepsia (11.8\%) and malabsorption (9\%). Almost $60 \%$ of procedures were performed on high definition endoscopes.

\section{Key endoscopic findings}

Sixty four UGN were detected of which 14 were dysplasias in the Barrett's esophagus, 1 Squamous intraepithelial neoplasia, 7 esophageal cancers, 3 esophageal neuroendocrine cancers, 8 gastric dysplastic lesions, 18 gastric cancers, 2 gastric neuroendocrine tumors, 9 duodenal adenomas and 2 duodenal cancers. CDR's components, i.e. esophageal inlet patch, gastric polyp, and duodenal bulb deformation were detected with rates of $5.8 \%, 15.3 \%$ and $2.6 \%$, respectively. Mean CDR was $21.9 \%$. In 1870 procedures, at least one biopsy was obtained, resulting in biopsy rate of $64.6 \%$ (see Table 1).

\section{Comparison of endoscopists}

Of 28 operators, 17 have performed at least 50 examinations (in total 2666 EGDs). The median CDR for analyzed operators was $17 \%$ and individual values ranged from 3.4 to $54 \%$. The operators were divided into four groups based on the CDR quartiles: group $1 \mathrm{CDR}<10 \%$ (4 operators), group 2 with CDR 10.0-17.0\% (5 operators), group 3 with CDR 17.1-26.0\% (4 operators) and group 4 with CDR > $26 \%$ (4 operators). UGN detection rate ranged from 0.5 to $7.1 \%$ for analyzed operators. Mean UGN detection values were $1.4 \%, 1.6 \%, 1.9 \%, 6 \%$ in groups $1-4$, respectively. In the logistic regression analysis, the odds ratio for neoplastic lesion detection increased from 1.1 to 4.4 in the groups $2-4$ 
Table 1 Demographic, endoscopic procedure and endoscopic findings data

\begin{tabular}{|c|c|}
\hline \multicolumn{2}{|l|}{ Demographic data } \\
\hline Number of patients & 2896 \\
\hline Mean age $(y \pm S D)$ & $56.9 \pm 16$ \\
\hline $\operatorname{Sex}(M / F)$ & $1237 / 1659$ \\
\hline \multicolumn{2}{|l|}{ Indication for endoscopy $[n / \%]$} \\
\hline Gastroesophageal reflux disease & $383 / 13.2 \%$ \\
\hline Dyspepsia & $343 / 11.8 \%$ \\
\hline Malabsorption & $261 / 9 \%$ \\
\hline Suspicion of malignancy & $221 / 7.6 \%$ \\
\hline Evaluation of portal hypertension & $175 / 6 \%$ \\
\hline Surveillance of gastritis & $167 / 5.8 \%$ \\
\hline Other & $1346 / 46.5 \%$ \\
\hline \multicolumn{2}{|l|}{ Procedure information } \\
\hline Sedation $[n / \%]$ & $968 / 32.4 \%$ \\
\hline High-definition/non-high-definition endoscopes & $1727 / 1169$ \\
\hline In/outpatient & $1075 / 1911$ \\
\hline \multicolumn{2}{|l|}{ Endoscopic findings $[n / \%]$} \\
\hline Gastric inlet patch & $168 / 5.8 \%$ \\
\hline Reflux esophagitis & $480 / 16.6 \%$ \\
\hline Non-dysplastic Barrett's esophagus & $70 / 2.4 \%$ \\
\hline Dysplasia in Barrett's esophagus & $14 / 0.5 \%$ \\
\hline Squamous intraepithelial neoplasia & $1 / 0.03 \%$ \\
\hline Esophageal cancer and neuroendocrine cancer & $10 / 0.4 \%$ \\
\hline Gastritis & $1991 / 68.8 \%$ \\
\hline Gastric polyps & $444 / 15.3 \%$ \\
\hline Gastric dysplastic lesions & $8 / 0.3 \%$ \\
\hline Gastric neuroendocrine tumors & $2 / 0.07 \%$ \\
\hline Gastric cancers & $18 / 0.6 \%$ \\
\hline Gastric peptic ulcer & $77 / 2.7 \%$ \\
\hline Helicobacter pylori infection & $289 / 10 \%$ \\
\hline Dudenal bulb deformation & $74 / 2.6 \%$ \\
\hline Duodenal ulcer & $55 / 1.9 \%$ \\
\hline Dudenal polyps & $44 / 1.5 \%$ \\
\hline Duodenal adenomas & $9 / 0.3 \%$ \\
\hline Duodenal cancer & $2 / 0.07 \%$ \\
\hline Helicobacter pylori infection/tested & $381 / 1486 / 25.6 \%$ \\
\hline \multicolumn{2}{|l|}{ Quality metrics $[n / \%]$} \\
\hline UGN & $64 / 2.2 \%$ \\
\hline Biopsy rate & $1870 / 64.6 \%$ \\
\hline CDR & $635 / 21.9 \%$ \\
\hline
\end{tabular}

$M$ Male, $F$ female, $H D$ high definition, CDR - composite detection rate, $U G N$ upper gastrointestinal neoplasm

in relation to group 1. The data are summarized in the Table 2. The correlation between CDR and UGN was statistically significant $(\mathrm{R}=0.49, p=0.045$; Fig. 2$)$.

\section{Risk factors of UGN detection}

In the univariate analysis, age, male gender, inpatient procedure, endoscopic center A (in comparison to center B) and EGD performed by endoscopist with CDR $>26 \%$ were risk factors of UGN detection. In the multivariate analysis, operators with CDR $>26 \%$ were over five times more likely to detect an UGN (OR 5.7; 95\% CI $1.5-22.3)$. Age and male gender were also found to be independent factors with—see Table 3 .

\section{Comparison of endoscopy centers}

Highest CDR was achieved in center A (35.3\%, university endoscopy center) and the highest biopsy rate (76.6\%) was recorded in center $\mathrm{C}$ (endoscopy unit of district hospital) in which CDR was the lowest (10.6\%). The differences in CDR and EBR were significant among centers. Overall UGN detection rate was $2.2 \%$ and was significantly higher in center $\mathrm{A}$ than in $\mathrm{B}$ and $\mathrm{C}(4.2 \%$ vs $1 \%$ vs $1.5 \%$, respectively; A vs $\mathrm{C}$ and $\mathrm{A}$ vs $\mathrm{B} p=$ both $<0.001$, B vs $\mathrm{C}$ $p=0.32$ ). The data are summarized in Table 4 .

\section{Relationship between EBR and CDR}

Mean EBR among operators was $64.6 \%$ ranging from 26.7 to $98.4 \%$. EBR was highest in the group with highest CDR, however, there was no clear correlation between either EBR and CDR ( $\mathrm{R}=0.36, p=0.16)$ or EBR and UGN detection $(\mathrm{R}=0.43, p=0.08)$. Threshold of $43.8 \%$ for EBR (validated by Januszewicz et al.) was fulfilled by 13 of 17 operators, however, reaching this threshold level insignificantly improved UGN detection (OR $1.55,95 \% \mathrm{CI}$ $0.8-3.1, p=0.18$ ) with detection rates $1.5 \%$ for $\mathrm{EBR}<43.8 \%$ vs $2.6 \%$ for $\mathrm{EBR} \geq 43.8 \%, p=0.2$ ).

\section{Discussion}

The appropriate quality indicator for EGD has been searched for some time with no tangible success. Difficulties in finding proper EGD quality indicator stem from several reasons. First, unlike in colonoscopy, which is targeted to detect and remove adenomatous polyps, in upper digestive tract the prevalence of precancerous lesions is too low to be treated as quality indicator [11]. Second, during the EGD, three different parts of digestive tract are investigated with different risk of cancer development and different phenotypes of the precancerous lesions. Generally, a quality indicator should be simple, easily analyzed among large numbers of endoscopies performed by different operators in different clinical settings. Several EGD quality metrics have been proposed so far. Procedure time [12-15] and 
Table 2 Operators performance based on composite detection rate

\begin{tabular}{|c|c|c|c|c|c|c|c|c|c|}
\hline Operator & Center & $\begin{array}{l}\text { CDR } \\
{[\%]}\end{array}$ & $\begin{array}{l}\text { UGN } \\
{[\%]}\end{array}$ & $\begin{array}{l}\text { EBR } \\
{[\%]}\end{array}$ & $\begin{array}{l}\text { CDR } \\
\text { group }\end{array}$ & $\begin{array}{l}\text { UGN } \\
{[\%]}\end{array}$ & OR for UGN detection $[95 \% \mathrm{CI}]$ & $p$ value & $\overline{\text { Biopsy rate }}$ \\
\hline 1 & A & $54.0 \%$ & $5.8 \%$ & $81.3 \%$ & Group 4 & $6.0 \%$ & $4.4[2.2-9.0]$ & $<0.001$ & $80.9 \%$ \\
\hline 2 & A & $42.8 \%$ & $4.8 \%$ & $89.7 \%$ & $>26 \%$ & & & & \\
\hline 3 & $\mathrm{~A}$ & $40.7 \%$ & $7.1 \%$ & $61.9 \%$ & & & & & \\
\hline 4 & A & $36.7 \%$ & $5.1 \%$ & $88.8 \%$ & & & & & \\
\hline 5 & $\mathrm{~B}$ & $25.7 \%$ & $0.8 \%$ & $51.3 \%$ & Group 3 & $1.9 \%$ & $1.2[0.5-2.8]$ & 0.67 & $54.7 \%$ \\
\hline 6 & $\mathrm{~B}$ & $24.8 \%$ & $1.5 \%$ & $64.9 \%$ & $17.1-26 \%$ & & & & \\
\hline 7 & $\mathrm{~B}$ & $17.8 \%$ & $1.8 \%$ & $31.4 \%$ & & & & & \\
\hline 8 & $\mathrm{C}$ & $17.5 \%$ & $4.8 \%$ & $98.4 \%$ & & & & & \\
\hline 9 & $\mathrm{~B}$ & $16.9 \%$ & $0.5 \%$ & $43.3 \%$ & Group 2 & $1.6 \%$ & $1.1[0.4-2.7]$ & 0.87 & $64.9 \%$ \\
\hline 10 & $\mathrm{C}$ & $16.1 \%$ & $1.8 \%$ & $78.6 \%$ & $10-17 \%$ & & & & \\
\hline 11 & $\mathrm{~A}$ & $15.4 \%$ & $1.5 \%$ & $53.8 \%$ & & & & & \\
\hline 12 & $\mathrm{C}$ & $12.6 \%$ & $2.2 \%$ & $74.8 \%$ & & & & & \\
\hline 13 & $\mathrm{C}$ & $10.5 \%$ & $1.9 \%$ & $93.3 \%$ & & & & & \\
\hline 14 & $\mathrm{C}$ & $9.9 \%$ & $0.5 \%$ & $83.3 \%$ & Group 1 & $1.4 \%$ & 1 & Reference & $60.6 \%$ \\
\hline 15 & B & $9.6 \%$ & $0.9 \%$ & $36.0 \%$ & $<10 \%$ & & & & \\
\hline 16 & $\mathrm{C}$ & $7.4 \%$ & $1.4 \%$ & $51.2 \%$ & & & & & \\
\hline 17 & A & $3.4 \%$ & $3.4 \%$ & $26.7 \%$ & & & & & \\
\hline
\end{tabular}

$C D R$ composite detection rate, $U G N$ upper gastrointestinal neoplasm, $E B R$ endoscopist biopsy rate, $O R$ odds ratio, $C I$ confidence interval

Fig. 2 Composite detection rate in relation to upper gastrointestinal neoplastic lesions detection. Each spot reflects an endoscopist. $C D R$ composite detection rate, $U G N$ upper gastrointestinal neoplasm

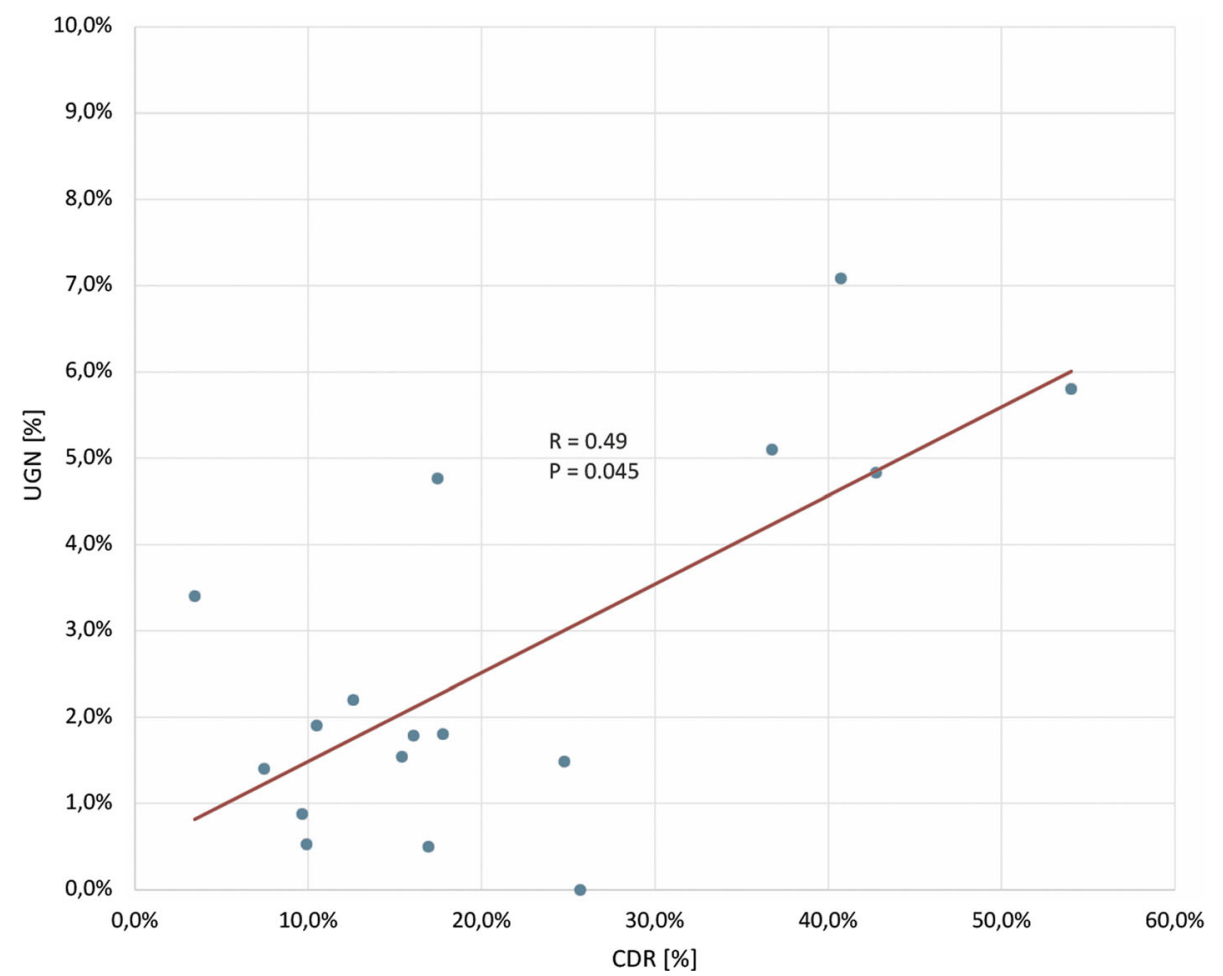

Vater's ampulla photo-documentation [16] have been recently proposed, however, low-quality data based on single-center investigations demand further validation. The most recently proposed quality metrics is endoscopist biopsy rate (EBR), which was shown to be related to reduced risk of missed gastric cancers [10]. However, there was no correlation between EBR and the detection of gastric dysplasias-conditions predisposing to cancer 
Table 3 Logistic regression analysis of upper gastrointestinal neoplasm detection risk factors

\begin{tabular}{|c|c|c|c|c|c|c|c|}
\hline \multirow[t]{2}{*}{ Variables } & \multirow{2}{*}{-} & \multicolumn{3}{|l|}{ Univariate } & \multicolumn{3}{|c|}{ Multivariate } \\
\hline & & OR & $95 \% \mathrm{CI}$ & $p$ value & OR & $95 \% \mathrm{CI}$ & $p$ value \\
\hline Age & & 1.03 & $1.01-1.05$ & $<0.001$ & $\underline{1.03}$ & $\underline{1.01-1.05}$ & $\underline{0.002}$ \\
\hline Male & & 2.0 & $1.2-3.4$ & 0.005 & $\underline{2.0}$ & $\underline{1.2-3.5}$ & $\underline{0.011}$ \\
\hline HD-endoscope & & 0.95 & $0.6-1.6$ & 0.858 & & & \\
\hline Sedation & & 0.8 & $0.4-1.3$ & 0.326 & & & \\
\hline Out-patient & & 0.4 & $0.2-0.7$ & $<0.001$ & $\underline{0.7}$ & $\underline{0.4-1.2}$ & $\underline{0.207}$ \\
\hline $\mathrm{CDR}>26 \%$ & & 4.6 & $2.8-8$ & $<0.001$ & $\underline{5.7}$ & $\underline{1.5-22.3}$ & $\underline{0.012}$ \\
\hline \multirow[t]{3}{*}{ Endoscopic center* } & $\underline{\mathbf{B}}$ & Reference & & & $\underline{0.8}$ & $\underline{0.3-2.0}$ & $\underline{0.666}$ \\
\hline & $\underline{\mathbf{C}}$ & $\underline{1.4}$ & $\underline{0.6}-3.1$ & $\underline{0.430}$ & & & \\
\hline & $\mathbf{A}$ & 4.0 & $2.0-8.1$ & $<0.001$ & & & \\
\hline
\end{tabular}

$H D$ high definition, CDR $>26 \%$ - examination performed by operator with CDR higher that $26 \%$ (group 4), OR - odds ratio, CI - confidence interval

*In the univariate analysis center with lowest mean UGN value (center B) was the reference. In the multivariate analysis the centers were graded according to mean UGN value - center B as 1 , center C as 2 and center $\mathrm{A}$ as 3
Table 4 Composite detection rate, biopsy rate and upper gastrointestinal neoplasm among centers

\begin{tabular}{llll}
\hline & Center A & Center B & Center C \\
\hline Patients [n] & 950 & 988 & 958 \\
Biopsy rate [\%] & $68.5 \%^{\mathrm{a}, \mathrm{b}}$ & $49.0 \%^{\mathrm{a}, \mathrm{c}}$ & $76.7 \%^{\mathrm{b}, \mathrm{c}}$ \\
CDR [\%] & $35.3 \%^{\mathrm{a}, \mathrm{b}}$ & $20.0 \%^{\mathrm{a}, \mathrm{c}}$ & $10.6 \%^{\mathrm{b}, \mathrm{c}}$ \\
UGN [n/\%] & $40 / 4.2 \%^{\mathrm{a}, \mathrm{b}}$ & $10 / 1 \%^{\mathrm{a}}$ & $14 / 1.5 \%^{\mathrm{b}}$ \\
\hline
\end{tabular}

$C D R$ composite detection rate, $U G N$ upper gastrointestinal neoplasm ${ }^{a}$ Significant difference in comparison center A with center B ${ }^{\mathrm{b}}$ Significant difference in comparison center A with center $\mathrm{C}$ ${ }^{\mathrm{c}}$ Significant difference in comparison center B with center C $p$-value $<0.017$ considered as significant according to Bonferroni's correction

development [17]. In our study, EBR value did not correlate with UGN detection either, suggesting that some endoscopists might be excessively focused on benign lesions with no clinical relevance. Other factors might also have impact on EBR. Routine biopsies to evaluate chronic gastritis, biopsies indicated by clinical background irrespective of endoscopic findings (for example in diagnosis of coeliac disease) increase EBR without reflection in neoplastic lesions detection. On the other hand, "no touch" optical diagnosis is also highly recommended to avoid fibrosis hindering radical endoscopic treatment. Financial aspect of EBR should also be raised. Pushing endoscopist to obtain a higher number of biopsy samples increases burden of pathomorphology departments with surge of procedure costs without obvious impact on clinical outcome. CDR based solely on endoscopic examination report is free of additional costs. Another phenomenon should also be mentioned. In the endoscopy unit where EBR was highest, the CDR was lowest with lower UGN detection than in the tertiary unit. It may suggest that biopsy without proper inspection does not protect against lesion omission.

Detection of precancerous conditions and early cancers is the one of the main goals of the EGD. Atrophic gastritis and gastric intestinal metaplasia detection rates were mentioned by Park et al. as EGD potential quality indicators, especially in the countries where gastric cancer prevalence is low [13]. However, their clinical significance is uncertain in low-risk gastric cancer populations [18, 19] and have not been validated as quality indicators. We assumed that the role of quality measure might be played by perception level of the endoscopist verified by his performance in finding tiny but more frequently occurring benign lesions. For this purpose, composite detection rate (CDR) was created based on 3 probably underreported endoscopic findings located in 3 different segments of the digestive tract explored in EGD, namely esophageal inlet patches, gastric polyps and duodenal bulb deformations. Esophageal inlet patches occur in general population with frequency of around $10 \%$, but are probably underrecognized [20]. Detection of these lesions were proposed as quality measure for EGD, however, no validation study for use of this parameter was done as yet [21]. It is noteworthy, that detection of tiny gastric polyps requires not only careful mucosal inspection but also thorough mucosal cleansing. Our assumption was that vigilant endoscopic inspection associated in the high detection of these 3 lesions is simultaneously associated with a higher number of detected neoplastic lesions. Therefore, the role of CDR was to measure general attentiveness and carefulness of endoscopist, which in a natural way improves ability to identify all kinds of pathologies within EGD reach. Similar 
idea of using gastric diverticula or subepithelial lesions has recently been put forward [11]. However, it seems that the prevalence of these lesions is too low to make them proper quality metric.

We prospectively validated CDR in three endoscopy units performing EGDs for in- and out- patients, localized in tertiary hospital, district hospital and private center. Such selection was aiming to cover the whole range of realworld endoscopy practice. The need for validation of quality indicators in different endoscopy units was also stressed by others [22]. Combination of lesion detection in the all parts of upper gastrointestinal tract seems to be appropriate as more than half of neoplastic lesions detected on EGD examinations were located in the esophagus or duodenum (36 out of 64 neoplastic lesions). The major finding of our study was positive correlation between CDR and neoplastic lesion detection rate. Based on our analysis we found that CDR of $26 \%$ is related to higher neoplastic lesions detection as well as being independent risk factor of neoplastic lesion detection in the upper gastrointestinal tract. This observation warrants further validation using CDR as quality indicator of EGD to clarify if it is correlated with improved detection of early cancers and lower rate of missed neoplasia. Analysis of missed neoplasm based on interval cancer development will be final confirmation of CDR effectiveness as well as opportunity to set the proper cutoff value.

We have found out that Helicobacter pylori prevalence in the study population was significantly lower than in the previous reports regarding Polish population [23]. It might reflect decreasing Helicobacter pylori prevalence in Poland stressed by others [24]. Also different testing methods, mass eradication effect, test and treat strategy in our study population, unknown status of previous infection treatment and used medications by enrolled patients may influence the lower infection rate.

Our study has several limitations. First, we did not compare CDR with other quality metrics such as examination time or Vater's ampulla photo-documentation [16]. Relation of CDR and mentioned metrics is an interesting point for the future research. Second, we were not able to validate $\mathrm{CDR}$ in the detection of only early lesions due to their low prevalence. Third, the performance of CDR may be different in the different populations such as countries with low prevalence of Helicobacter pylori infection (lower incidence of gastritis and duodenitis), low PPI intake rate or screening population. Finally, referral bias was not taken into account in our study. UGN detection rate was the highest in the tertiary center. UGN detection might be higher due to possible patients preselection which could result in higher number of patients with precancerous conditions of high neoplastic lesions development risk (e.g., long Barrett's esophagus, extensive atrophic gastritis).

In conclusion, CDR is promising quality indicator of EGD showing close relationship with detection of neoplastic lesions.

Acknowledgements The first author would like to thank Marek Bugajski, Katarzyna Pawlak and Bettina Golec for reviewing the manuscript.

Funding This research received no specific funding including grant support.

\section{Declarations}

Conflict of interest The authors declare that they have no conflict of interest.

Open Access This article is licensed under a Creative Commons Attribution 4.0 International License, which permits use, sharing, adaptation, distribution and reproduction in any medium or format, as long as you give appropriate credit to the original author(s) and the source, provide a link to the Creative Commons licence, and indicate if changes were made. The images or other third party material in this article are included in the article's Creative Commons licence, unless indicated otherwise in a credit line to the material. If material is not included in the article's Creative Commons licence and your intended use is not permitted by statutory regulation or exceeds the permitted use, you will need to obtain permission directly from the copyright holder. To view a copy of this licence, visit http://creativecommons. org/licenses/by/4.0/.

\section{References}

1. Peery AF, Crockett SD, Murphy CC, et al. Burden and cost of gastrointestinal, liver, and pancreatic diseases in the United States: update 2018. Gastroenterology. 2020;156:254-72.

2. Bray F, Ferlay J, Soerjomataram I, et al. Global cancer statistics 2018: GLOBOCAN estimates of incidence and mortality worldwide for 36 cancers in 185 countries. CA Cancer J Clin. 2018;68:394-424.

3. Jun JK, Choi KS, Lee HY, et al. Effectiveness of the Korean National Cancer Screening Program in reducing gastric cancer mortality. Gastroenterology. 2017;152:1319-28.

4. Beg S, Ragunath K, Wyman A, et al. Quality standards in upper gastrointestinal endoscopy: a position statement of the British Society of Gastroenterology (BSG) and Association of Upper Gastrointestinal Surgeons of Great Britain and Ireland (AUGIS). Gut. 2017;66:1886-99.

5. Bisschops R, Areia M, Coron E, et al. Performance measures for upper gastrointestinal endoscopy: a European Society of Gastrointestinal Endoscopy (ESGE) Quality Improvement Initiative. Endoscopy. 2016;48:843-64.

6. Park WG, Shaheen NJ, Cohen J, et al. Quality indicators for EGD. Gastrointest Endosc. 2015;81:17-30.

7. Pimenta-Melo AR, Monteiro-Soares M, Libânio D, et al. Missing rate for gastric cancer during upper gastrointestinal endoscopy: a systematic review and meta-analysis. Eur J Gastroenterol Hepatol. 2016;28:1041-9.

8. Rodríguez de Santiago E, Hernanz N, Marcos-Prieto HM, et al. Rate of missed oesophageal cancer at routine endoscopy and 
survival outcomes: a multicentric cohort study. United Eur Gastroenterol J. 2019; 7:189-198.

9. Kaminski MF, Thomas-Gibson S, Bugajski M, et al. Performance measures for lower gastrointestinal endoscopy: a European Society of Gastrointestinal Endoscopy (ESGE) quality improvement initiative. United Eur Gastroenterol J. 2017;5:309-34.

10. Januszewicz W, Wieszczy P, Bialek A, et al. Endoscopist biopsy rate as a quality indicator for outpatient gastroscopy: a multicenter cohort study with validation. Gastrointest Endosc. 2019;89:1141-9.

11. Park CH, Kim B, Chung H, et al. Endoscopic quality indicators for esophagogastroduodenoscopy in gastric cancer screening. Dig Dis Sci. 2014;60:38-46.

12. Teh JL, Tan JR, Lau LJF, et al. Longer examination time improves detection of gastric cancer during diagnostic upper gastrointestinal endoscopy. Clin Gastroenterol Hepatol. 2015;13:480-7.

13. Park JM, Huo SM, Lee HH, et al. Longer observation time increases proportion of neoplasms detected by esophagogastroduodenoscopy. Gastroenteorlogy. 2017;153:460-9.

14. Kawamura T, Wada H, Sakiyama N, et al. Examination time as a quality indicator of screening upper gastrointestinal endoscopy for asymptomatic examinees. Dig Endosc. 2017;29:569-75.

15. Gupta N, Gaddam S, Wani SB, et al. Longer inspection time is associated with increased detection of high-grade dysplasia and esophageal adenocarcinoma in Barrett's esophagus. Gastrointest Endosc. 2012;76:531-8.

16. Park JM, Lim CH, Cho YK, et al. The effect of photo-documentation of the ampulla on neoplasm detection rate during esophagogastroduodenoscopy. Endoscopy. 2019;51:115-24.

17. Song H, Ekheden IG, Zheng Z, et al. Incidence of gastric cancer among patients with gastric precancerous lesions: observational cohort study in a low risk Western population. BMJ. 2015;351:h3867.

18. Pimentel-Nunes P, Libânio D, Marcos-Pinto R, et al. Management of epithelial precancerous conditions and lesions in the stomach (MAPS II): European Society of Gastrointestinal Endoscopy (ESGE), European Helicobacter and Microbiota Study Group (EHMSG), European Society of Pathology (ESP), and Sociedade Port. Endoscopy. 2019;51:365-88.

19. Gupta S, Li D, El Serag HB, et al. AGA clinical practice guidelines on management of gastric intestinal metaplasia. Gastroenterology. 2020;158:693-702.

20. Rusu R, Ishaq S, Wong T, et al. Cervical inlet patch: new insights into diagnosis and endoscopic therapy. Frontline Gastroenterol. 2018;9:214-20.

21. Bajbouj M, Meining A, Schmid RM. Endoscopic diagnosis and treatment of inlet patch: justification, techniques, and results. Tech Gastrointest Endosc. 2014;16:49-52.

22. Min JK, Cha JM, Kwak MS, et al. Quality indicators and outcome measures of endoscopy in the national cancer screening program. Yonsei Med J. 2019;60:1054-60.

23. Hooi JKY, Lai WY, Ng WK, et al. Global prevalence of Helicobacter pylori infection: systematic review and meta-analysis. Gastroenterology. 2017;153:420-9.

24. Tacikowski T, Bawa S, Gajewska D, et al. Current prevalence of Helicobacter pylori infection in patients with dyspepsia treated in Warsaw. Poland Prz Gastroenterol. 2017;12:135-9.

Publisher's Note Springer Nature remains neutral with regard to jurisdictional claims in published maps and institutional affiliations. 